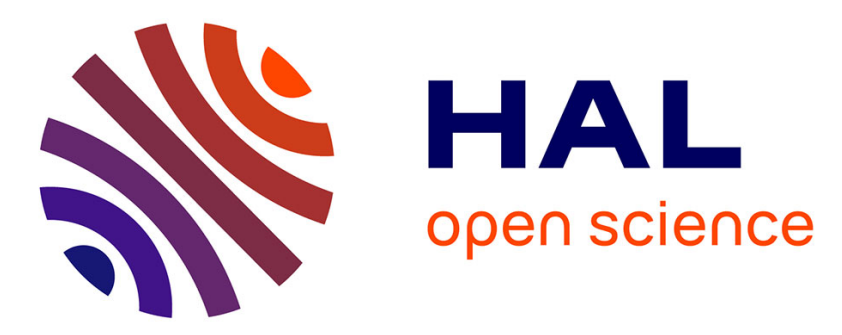

\title{
Absolute stability of a Bénard-von Kármán vortex street in a confined geometry
}

\author{
Paul Boniface, Luc Lebon, Laurent Limat, Mathieu Receveur
}

\section{To cite this version:}

Paul Boniface, Luc Lebon, Laurent Limat, Mathieu Receveur. Absolute stability of a Bénard-von Kármán vortex street in a confined geometry. EPL - Europhysics Letters, 2017, 117 (3), pp.34001. 10.1209/0295-5075/117/34001 . hal-02343763

\section{HAL Id: hal-02343763 https://hal.science/hal-02343763}

Submitted on 3 Nov 2019

HAL is a multi-disciplinary open access archive for the deposit and dissemination of scientific research documents, whether they are published or not. The documents may come from teaching and research institutions in France or abroad, or from public or private research centers.
L'archive ouverte pluridisciplinaire HAL, est destinée au dépôt et à la diffusion de documents scientifiques de niveau recherche, publiés ou non, émanant des établissements d'enseignement et de recherche français ou étrangers, des laboratoires publics ou privés. 


\title{
Absolute stability of a Bénard-von Kármán vortex street in a con- fined geometry
}

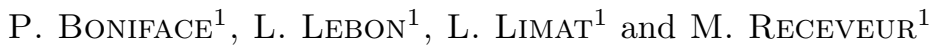 \\ 1 Matière et Systèmes Complexes, CNRS and Université Paris Diderot UMR 7057, Bâtiment Condorcet, 10 rue Alice \\ Domon et Léonie Duquet, 75013 Paris, France
}

PACS $47.20 . \mathrm{Ft}$ - Instability of shear flows

PACS $47.32 . \mathrm{ck}$ - Vortex streets

PACS $47.20 .-\mathrm{k}-$ Flow instabilities

\begin{abstract}
We have investigated the stability of a double vortex street, induced in a rectangular container by a tape, or a rope, moving at high speed on its free surface. Depending on the tape velocity and on the geometrical aspect ratios, three patterns of flows are observed: (1) a vortex street with recirculation of the liquid along the lateral sides of the container, (2) the same recirculation but with no stable vortex array, (3) recirculation along the bottom of the container. We have investigated the spatial structure of the vortex street and found that this system explores the phase space available inside a stability tongue predicted at the end of the 1920 s by Rosenhead for point vortices in a perfect fluid. Although this very surprising result contrasts with the wellknown von Kármán unique stability condition for point vortex streets in an infinite domain, this complements the theory inside a channel of finite breadth. In this paper, we present the very first experimental confirmation of this 90 -year old theory.
\end{abstract}

The Bénard-Von Kármán (BVK) vortex street is a structure that can be observed in the wake of an obstacle immersed inside a stationary unidirectional flow of large enough velocity [1]. It was investigated experimentally for the first time by Bénard [2] and modeled by von Kármán [3] [4] at the beginning of the XXth century and has since been extensively investigated. This phenomenon is ubiquitous in meteorology, oceanography, naval engineering, vehicle design, modeling of the air flows around buildings or piles of bridges, etc [5]. According to Von Kármán [3], a vortex street of point vortices in a plane perfect flow can be stable only if the wavelength of the vortex street, i.e. the distance between two consecutive vortices in one array of the street, $2 b$, is linked to the width of the street $2 a$ by

$$
\frac{a}{b}=\frac{1}{\pi} \operatorname{argcosh}(\sqrt{2}) \approx 0.281
$$

Though this condition is very well satisfied in experiments, theoretically, the precise nature of this unique stable solution, is still under discussion after several controversies [6] [7] [8] [9]. Recently, it has been proposed that the stability could be in fact only apparent and of convective nature, in the sense of Huerre, Chomaz and
Monkewitz [10]. The whole vortex arrangement would be in fact intrinsically unstable in its own framework [11].

A very different situation arises when the bidimensional vortex street is confined between two lateral walls, separated by a distance $2 c$. Calculations performed on point vortices in the complex plane addressed this question at the end of the 20's, and found non trivial results [12] [13] [14] [15]. In particular, Rosenhead [13], established that in this configuration, equation (1) had to be replaced in the plane $(a / c, b / c)$ by a continuous stability tongue of finite and non-zero extent. To our knowledge, no experimental check of this result has been published yet, though recent attempts combined with numerical computations have now begun to appear [16] [17]. Experimental investigations of this phenomenon in this configuration is difficult : usual experiments combine the vortex emission behind the obstacle with the wake growth and reorganization downstream. This growth involves complex questions about the absolute and convective nature of the vortex system stability [18] [19]. 

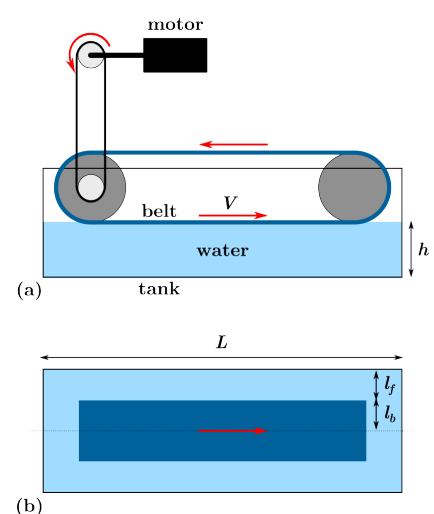

Fig. 1: (a) and (b), detailed stretch of our experiment, in which a belt (tape) runs at high speed along the central part of the free surface of a long slender tank of water. In (c) and (d), are represented the two main patterns of flow observed at large scale: in (c) the recirculation occurs at the bottom of the container and in (d) it occurs on the lateral sides.
In this letter, we present a new experimental study using a completely different strategy. We present a new method to create double rows of vortices with almost no mean velocity ; their stability can be investigated at will for various degrees of confinement [20] [21]. We induce a longitudinal flow inside a long rectangular pool using a tape moving at high speed on the free surface of a layer of water. The liquid below the tape is dragged at the tape velocity, and a recirculation has to occur in the opposite direction because of mass conservation. If this recirculation occurs on the lateral sides of the tank, the mixing layers between the two flows can become unstable and two coupled Kelvin-Helmholtz instabilities develop [22] [23], leading to two counter-rotative vortex rows spatially out of phase, i.e a classic vortex street. The spatial properties of this street can be investigated varying the degree of confinement, using moving lateral walls and varying the water layer depth. Because of mass conservation the mean velocity calculated across a section of the container vanishes which implies that the double row of vortices is studied nearly in its own frame without any mean flow superimposed to it, in absolute stability conditions. This flow geometry is intrinsically of great interest, as it can be viewed as an uncurved equivalent of Couder et al's experiments [24] in which vortices were formed and observed in the vicinity of concentric rotating disks. Both geometries have a great technical interest for applications in which a thin plate or a disk is moving at high speed between static walls as it is the case for moving belts [25] and hard disks drives [26] [27]. Our geometry has also, presumably, some implications for the drag resistance of slender boats advancing in a narrow channel.

The experiment is represented on figure 1. An endless tape of width $2 l_{b}$ is pulled with a constant speed at the
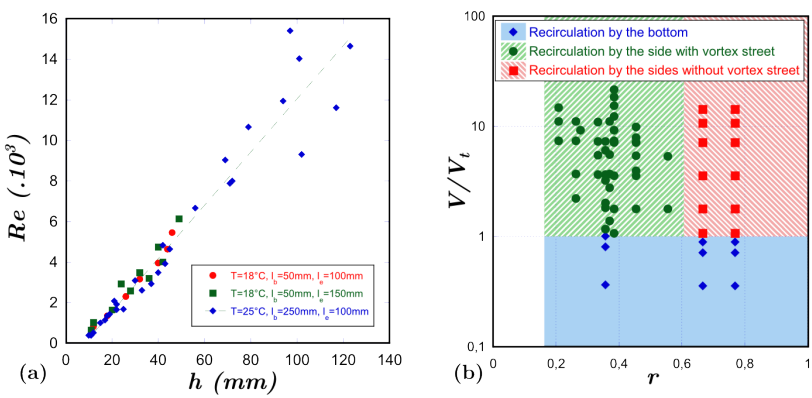

(c)

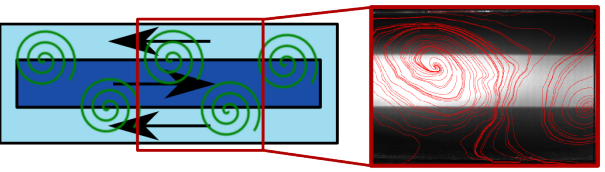

(d)

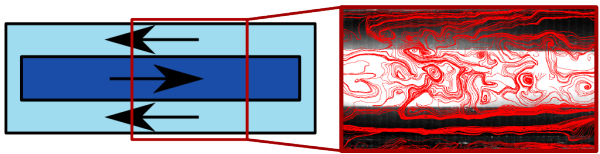

Fig. 2: (a) Experimental measurement of the Reynolds number at which we observe a transition from the recirculation by the bottom flow to the lateral sides. (b) Phase diagram of the three flow patterns observed for $h=45 \mathrm{~mm}$ : recirculation by the bottom (blue), recirculation along the lateral walls with double vortex row formation (green), recirculation along the lateral walls with no stable array (red). (c) and (d) show typical instantaneous flow lines observed in the two last cases.

free surface of a liquid layer in a long pool, of length $L=2 \mathrm{~m}$ and with a rectangular cross section : $70 \mathrm{~cm}$ in width versus $20 \mathrm{~cm}$ in height. The tape velocity $V$ can vary between 1 to $140 \mathrm{~cm} \cdot \mathrm{s}^{-1}$. It is possible to select smaller lateral length, $2 l_{b}<2 c<70 \mathrm{~cm}$, by using adjustable walls, while the liquid depth $h$ can be selected at will by removing or adding water before the experiment. Two tape widths have been explored: $l_{b}=25$ $\mathrm{cm}$ and $l_{b}=5 \mathrm{~cm}$. To investigate the effects of a very thin belt, we also used an endless rope with a diameter of $0.5 \mathrm{~cm}$. The container is made of transparent glass, in order to allow us to use optical diagnostics and in particular particle image velocimetry (PIV) ; Polyamide seeding particles (PSP) with a diameter of $50 \mu \mathrm{m}$ were dispersed in the liquid before measurements, and their motion was followed by a tunable laser sheet and a high speed video camera. The particles' motion is analyzed and gives the velocity field, its instantaneous streamlines or the vorticity distribution in a selected plan.

Depending on the tape velocity and on the aspect ratios, three very different kinds of flow have been observed. The liquid immediately below the tape is dragged in one direction, while mass conservation inside a closed channel implies that the liquid must somewhere recirculate in the opposite direction. At low tape velocity, all the free surface follows the tape motion, while the liquid recircu- 

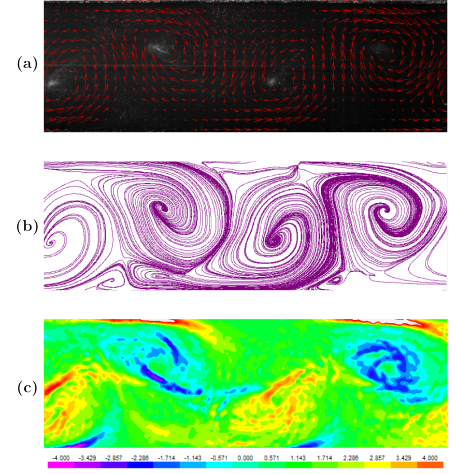

Fig. 3: Typical flow observed in a horizontal plane, at the middle height of the water layer inside the container, using a rope instead of the belt. As the vortex street does not move, these are temporal means calculated over 30 seconds. (a) fluid velocity calculated by PIV, (b) stream lines, (c) vorticity distribution.

lates by the bottom of the container (figure 1.c). Above a critical velocity, the flow structure changes drastically: the recirculation occurs mainly along the channel walls (figure 1.d). A layer of high shear develops between the flow directly dragged by the tape and the flow that follows the lateral container walls, which gives rise to eddy formation via the famous Kelvin-Helmholtz instability. Depending on the aspect ratios of the geometrical lengths involved $\left(2 l_{b}, h, 2 c\right)$, this eddy formation can either give rise to a uniform turbulent state represented in figure 2.d, in which vortices of all sizes are observed with complex behaviors (coalescence, appearance, disappearance...), or to a coherent state represented in figure 2.c with large structures adopting the geometry of a BVK vortex street, each row being placed on other side of the floating tape in spatial phase opposition with each other. Beautiful streets were also obtained with a rope pulled at the free surface of the container (figure 3) instead of a belt, but in this case, only this kind of flow was observed in our experimental conditions. In all the cases explored, the vortex streets were static or nearly static in the framework of the container. The drift velocity was exactly equal to zero for the experiments with the smallest values of $l_{b}$ (and hence with a rope), while a slight but noticeable drift in the direction opposite to the tape motion was noticed in the belt case, with a maximal velocity smaller than $8 \%$ of the tape velocity.

The critical velocity $V_{c}$ at which the recirculation flow ceases to occur at the bottom and starts around the lateral walls of the container has been measured as a function of liquid depth $h$ for two different tape widths, $l_{b}=25$ $\mathrm{cm}$ and $l_{b}=5 \mathrm{~cm}$, and two different lateral confinements, $l_{f}=c-l_{b}=10 \mathrm{~cm}$ and $15 \mathrm{~cm}$. The results are plotted on figure 2.a, in which we have defined the Reynolds number as $R e=\frac{h V}{\nu}$. Slight variations of temperatures have been taken into account in the estimation of the $\nu$ value [28].
All the data seem to collapse on a single straight line, which implies that the Reynolds at the transition $R e_{t}$ only depends on the depth of the water $\mathrm{h}$, and is governed by the following empirical law

$$
R e_{t}=A h-B .
$$

with $A \approx 1.3 \times 10^{5} \mathrm{~m}^{-1}$ and $B \approx 950$. This corresponds to a belt velocity at the transition $V_{t}=\nu\left(A-\frac{B}{h}\right)$ that reaches empirically $V_{t}(h \rightarrow \infty)=13 \mathrm{~cm} \cdot \mathrm{s}^{-1}$, at $20^{\circ} \mathrm{C}$ in water. The order of magnitude of $V_{t}$ suggests a link with capillarity-gravity wave drag at the free surface [29]. This could explain how the tape can entrain so easily the whole free surface in a certain range of velocity. Having however scaled the tape velocity on this empirical scale, we have built phase diagrams specifying when each of the three flows were observed. A typical example is reproduced on figure 2.b, in which the ratio $r=l_{b} / c$ plotted on the horizontal axis measures the degree of confinement applied to the obtained array, as most often the distance between each rows seemed to be close to $l_{b}$. It appears that the double row of vortices is unstable when the confinement is high, but becomes stabilized in its own frame, when the confinement is sufficiently low. These observations are consistent with the ideas presented recently that the double row is in fact intrinsically unstable in an absolute frame, but can be stabilized apparently by convective effects, or can be really absolutely stabilized by the action of walls confining laterally the flow [11].

The precise geometry selection of the vortex street is a complex question. As suggested on figure 4-a, the system can chose two typical lengths : the width of the street $2 a$ and its wavelenght $2 b$. We have investigated the $a$ and $b$ dependence upon $l_{b}$ and $c$. The experimental values are highly fluctuating in time, and so is the residual drift velocity of the street. In this paper we will only focus on the possible correlation between $a$ and $b$ that should replace equation (1) in a confined geometry.

The stability of a vortex street in a two-dimensionnal channel was extensively investigated at the end of the 1920 s, in the framework of point vortices in the complex plane. The complex potential of the flow can be calculated exactly in the base state by adding the velocity flows induced by the two rows of vortices and all their successive images by the walls ; this guarantees the boundary condition of a vanishing normal velocity at the walls. Following Rosenhead [13], the complex potential reads:

$$
w(z=x+i y)=-\frac{i \kappa}{2 \pi} \log \left[\frac{\vartheta_{1}\left(\frac{z}{2 b}-i \frac{a}{2 b} \mid \frac{2 i c}{b}\right) \vartheta_{3}\left(\frac{z}{2 b}-i \frac{a}{2 b} \mid \frac{2 i c}{b}\right)}{\vartheta_{2}\left(\frac{z}{2 b}+i \frac{a}{2 b} \mid \frac{2 i c}{b}\right) \vartheta_{4}\left(\frac{z}{2 b}+i \frac{a}{2 b} \mid \frac{2 i c}{b}\right)}\right]
$$

where $x$ is the streamwise coordinate, $y$ the transverse coordinate and $\kappa$ the circulation of the vortex. The functions $\vartheta_{i}(u, \tau)$ are the elliptic functions of Tannery and Molk [30], that read, for $q=e^{i \pi \tau}$ : 


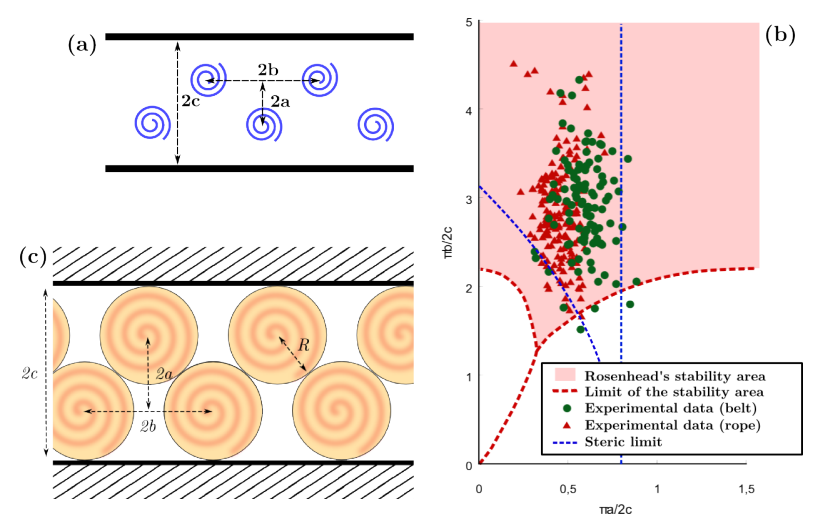

Fig. 4: (a) Sketch of the vortex street with the definition of the variables $\mathrm{a}, \mathrm{b}$ and $\mathrm{c}$. (b) Sketch of the vortex street with maximal density, when the vortex are modelized as rigid disks with radius R. (c) Representation of the stability area calculated by Rosenhead and the geometric limits given by the rigid disks model. The lower part of stability zone, when $1 / c$ tend to 0 , coincides with von Kármán condition giver by equation (1), but grows progressively at larger values of the $b / c$ ratio. We have plotted all our experimental data, with the rope setup or the belt setup. It appears that the stability area predicted by Rosenhead, inside the geometric limitations, is explored and satisfied by the experimental measures.

$$
\begin{aligned}
& \text { - } \vartheta_{1}(u \mid \tau)=2 \sum_{n}(-1)^{n} q^{\left(n+\frac{1}{2}\right)^{2}} \sin ((2 n+1) \pi u), \\
& \text { - } \vartheta_{2}(u \mid \tau)=2 \sum_{n} q^{\left(n+\frac{1}{2}\right)^{2}} \cos ((2 n+1) \pi u), \\
& \text { - } \vartheta_{3}(u \mid \tau)=1+2 \sum_{n} q^{n^{2}} \cos (2 \pi n u), \\
& \text { - } \vartheta_{4}(u \mid \tau)=1+2 \sum_{n}(-1)^{n} q^{n^{2}} \cos (2 \pi n u) .
\end{aligned}
$$

Rosenhead investigated the stability of the vortex street, having the potential of equation (3). He found that this system was stable in a tongue of finite extent, in the plane $(a / c, b / c)$ that is presented on figure 4.c. We plotted our fluctuating values of $a$ and $b$, for stable vortex streets obtained with a belt and a rope, on figure 4.c. All the data are dispersed inside the Rosenhead stability area and are not accumulating on the von Kármán limit given by equation (1). This remarkable result indicates that the presence of the walls modifies the stability of the street, allowing for a continuous band of ratios $b / a$, instead of the "sharp" selection observed on isolated wakes. As mentioned above, a particularity of our experiment is that we are in conditions of zero mean velocity superimposed to the array, which implies that our result holds for the absolute stability of the double row.

Another surprising result when one considers figure 4.c, is that though the points are distributed in a continuous way inside Rosenhead stability tongue, they do not cover the whole allowed domain and remain distributed in a rather narrow band of $a / c$ values. We suggest that this could partly be due to the finite extent of the vortex cores, which is of course not captured by Rosenhead point vortex model. Up to now, there is no equivalent of Rosenhead calculations for vortices of finite size, but we were able to develop here the following qualitative argument, based on the structure suggested on figure 4.b. If we consider the vortex cores as rigid disks that must tile the available space without overlapping, but keeping contacts between each other and with the two boundaries, it is easy to show that the ratios $\mathrm{a} / \mathrm{c}$ and $\mathrm{b} / \mathrm{c}$ must satisfy the following relationships

$$
\begin{gathered}
\frac{b}{c}>2 \sqrt{1-2 \frac{a}{c}}, \\
\frac{a}{c}<\frac{1}{2} .
\end{gathered}
$$

We have indicated these two limits on figure 4.c, that reduce the available part of the stability tongue and seem to explain remarkably well the observed confinement of the points inside a narrow band of $a / c$ values. There is clearly something to understand related to this question of finite size of vortex cores, but the extension of Rosenhead calculations to this situation is certainly a difficult task. At this stage, detailed analysis of the finite size of vortices only exist in the case of non confined geometry [31].

To conclude, we have carried out a new experiment to study the absolute stability of a BVK street in its own frame. In contrast with the well-known selection given by (1), we observed that the system explores a continuous band of stability, in a way consistent with Rosenhead's calculations. These observations are presumably the first and presently the unique experimental confirmation of this theory. We have also provided evidences that suggest reconsidering this theory with a finite size of the vortex cores, which is certainly a difficult theoretical challenge. It would be also interesting to check what happens for a wake behind an obstacle confined between two walls. The present result is presumably important for the diverse applications mentioned at the beginning of the paper, and for the drag encountered by a moving slender boat inside a narrow channel, the momentum carried by the vortices being to be added to the more classical wave drag due to surface waves [29].

$$
* * *
$$

We are indebted to stimulating discussions with C. Arratia, J.-M. Chomaz, P. Ern, F. Gallaire, C. Gondret, C.T. Pham, M. Rabaud, L. Tuckerman and J. E. Wesfreid.

\section{REFERENCES}

[1] Provansal M., Mathis C. and Boyer L., J. Fluid Mech., 182 (1987) 1.

[2] Bénard H., Comptes Rendus de l'Academie des Sciences, . (1908) 970. 
[3] VON KARMAN T., Nachrichten von der Gesellschaft der Wissenschaften zu Göttingen, MathematischPhysikalische Klasse, 1911 (1911) 509.

[4] von Karman T. and Rubach H., Phys. Z, 13 (1912) 49.

[5] Marris A. W., J. Fluids Eng., 86(2) (1964) 185.

[6] Saffman P. and Schatzman J., J. Fluid Mech., 117 (1982) 171.

[7] Kida S., J. Fluid Mech., 122 (1982) 487.

[8] Meiron D., Saffman P. and Schatzman J., J. Fluid Mech., 147 (1984) 187.

[9] Jimenez J., J. Fluid Mech., 178 (1987) 177.

[10] Huerre P. and Monkewitz P., Annu. Rev. Fluid. Mech., 22 (1990) 473.

[11] Mowlavi S., Arratia C. and Gallaire F., Journal of Fluid Mechanics, 795 (2016) 187.

[12] Glauert H., Proc. Roy. Soc. Lond. A, 120 (1928) 34.

[13] Rosenhead L., Philos. Trans. Roy. Soc. London, 228 (1929) 275.

[14] Tomotika S., Proceedings of the Physico-Mathematical Society of Japan, 48 (1929) 214.

[15] Villat H., Ann. Sci. E.N.S 3e série, 46 (1929) 259.

[16] Rees S. J. and Juniper M. P., J. Fluid Mech., 656 (2010) 309 .

[17] Biancofiore L. and Gallaire F., Phys. Fluids, 23 (2011) 034103

[18] Chomaz J. M., Rabaud M., Basdevant C. and Couder Y., J. Fluid Mech., 187 (1988) 115.

[19] Goujon-Durand S., Jenfer P. and Wesfreid J., Physical Review E, 50(1) (1994) 308.

[20] BONIFACE P., Instabilité de kelvin-helmholtz et allée de bénard-von kármán en géométrie rectangulaire confinée Ph.D. thesis Université Paris Diderot-Paris 7 (2014).

[21] Boniface P., Lebon L., Boulllet F., Receveur M. and LimAT L., Stabilité absolue d'une allée de bénard-von kármán confinée engendrée par deux instabilités couplées de kelvin-helmholtz presented at Comptes-endus de la 17 e Rencontre du Non-Linéaire 2014.

[22] Chandrasekhar S., Hydrodynamic and hydomagnetic stability (Oxford University) 1961.

[23] Huerre P. and Rossi M., Hydrodynamics and non-linear instabilities (Cambridge University Press) 1998 Ch. Hydrodynamic instabilities in open flows pp. 81-288.

[24] Rabaud M. and Couder Y., J. Fluid Mech., 136 (1983) 291.

[25] Chang Y., Fox S., Lilley D. and Moretti P., Machinery Dynamics and Element Vibrations ASME, 36 (1991)

[26] Kirpekar S. and Bogy D., Magnetics, IEEE Tansactions on, 42 (2006) 1716.

[27] Ng E., LiU N. and TAN Y., The Open Numerical Methods Journal, 3 (2011) 31.

[28] Weast R., Astle M. and Beyer W., CRC handbook of chemistry and physics (CRC press Boca Raton, FL) 1988.

[29] Le Merrer M., Clanet C., Quéré D., Raphä̈l E. and Chevy F., Proc. Natl. Acad. Sci. U. S. A., 108 (2011) 15064.

[30] Tannery J., Molk J. and Hermite C., Éléments de la théorie des fonctions elliptiques (vol.1 - 4) (GauthierVillars) 1893.

[31] Crowdy D. and Green C., Phys. Fluids, 23 (2011) 126602. 